\title{
Transcription factor 7-like 2 polymorphisms and diabetic retinopathy: a systematic review
}

\author{
P. Sudchada ${ }^{1,2}$ and K. Scarpace ${ }^{2}$ \\ ${ }^{1}$ Department of Pharmacy Practice, Faculty of Pharmaceutical Sciences, \\ Naresuan University, Phitsanulok, Thailand \\ ${ }^{2}$ School of Pharmacy, University of Wisconsin-Madison, Madison, WI, USA \\ Corresponding author: P. Sudchada \\ E-mail: psudchada@gmail.com \\ Genet. Mol. Res. 13 (3): 5865-5872 (2014) \\ Received November 21, 2013 \\ Accepted February 18, 2014 \\ Published August 7, 2014 \\ DOI http://dx.doi.org/10.4238/2014.August.7.1
}

ABSTRACT. The global prevalence of type 2 diabetes mellitus (T2DM) has increased, as well as complications including diabetic retinopathy. Polymorphisms in transcription factor 7-like 2 (TCF7L2) have been associated with T2DM, with the strongest association attributed to the single-nucleotide polymorphism rs7903146. In this review, we searched the current literature to determine whether an association exists between TCF7L2 polymorphisms rs7903146 with diabetic retinopathy. A systematic search was performed of EMBASE, PubMed, and Scopus using the following search terms: diabetic, retinopathy, polymorphism, genetic, transcription factor 7-like 2, TCF7L2. A manual search was also performed. There was no language or study design restriction. Three full articles and one abstract were reviewed. All studies were retrospective case-control studies that compared the frequency of the wild-type $\mathrm{CC}$ genotype and genotypes with the risk $\mathrm{T}$ allele. None of the studies found a statistically significant odds ratio. While the number of studies examined was small, this review suggests that there is no risk of diabetic retinopathy among individuals with the TCF7L2 polymorphisms rs7903146; however, the polymorphism may play a small role in diabetic retinopathy. Future prospective studies and trials involving diverse ethnicities that adjust for confounding 
variables are required to understand the association between TCF7L2 polymorphisms and diabetic retinopathy.

Key words: Diabetic retinopathy; Systematic review; TCF7L2; Transcription factor 7-like 2 polymorphisms

\section{INTRODUCTION}

The prevalence of type 2 diabetes mellitus (T2DM) has increased in recent decades. The current global prevalence is estimated to be $6 \%$ and it is projected that 300 million people will be affected by 2025 (Zimmet et al., 2001; Tong et al., 2009; Yau et al., 2012). Among its clinical outcomes, diabetes microvasculature complications threaten the quality of life for many patients. Diabetic retinopathy is one of the leading causes of blindness worldwide in middle-aged adults. The global prevalence of diabetic retinopathy was estimated to be $34.6 \%$ through the evaluation of 22,896 diabetic patients in 35 studies, corresponding to 93 million patients living with diabetic retinopathy in 2010 (Yau et al., 2012).

A specific gene, transcription factor 7-like 2 (TCF7L2), has reproducibly demonstrated to be associated with T2DM (Tong et al., 2009). The TCF7L2 gene is located on chromosome 10 and encodes for a transcription factor involved in the Wnt signaling pathway (Lyssenko, 2008). When the Wnt signaling pathway is inactivated, a complex involving TCF7L2 represses Wnt gene expression. When activated, TCF $7 L 2$ forms a complex with $\beta$-catenin to activate expression of the Wnt signaling gene. This pathway is vital for regulating cell morphology, proliferation, motility, oncogenesis, and tumor suppression (Lyssenko, 2008; Hansson et al., 2010).

The association between 2 specific TCF7L2 polymorphisms, rs12255372 and rs7903146, and T2DM was first reported in 2006 (Grant et al., 2006). The strongest association with T2DM was identified as a single-nucleotide polymorphism (SNP) in intron 4, rs7903146 (Helgason et al., 2007; Weedon, 2007; Lyssenko, 2008), which replaces cytosine with thymine and the risk allele identified as $\mathrm{T}$ (Cauchi and Froguel, 2008). The risk T allele has been associated with impaired insulin secretion, insulin processing, and incretin effects, subsequently resulting in hyperglycemia (Grant et al., 2006; Loos et al., 2007, Jin and Liu, 2008; Lyssenko, 2008; Shu et al., 2008; Hansson et al., 2010). In vitro studies have demonstrated that $T C F 7 L 2$ is required for pancreas development through active Wnt signaling and cytoprotection of $\beta$ cells (Lyssenko, 2008). When the TCF7L2 gene was knocked down in vitro in isolated islets, decreased $\beta$-cell survival and functioning were observed (Shu et al., 2008; Hansson et al., 2010). TCF7L2 also has binding sites on the proglucagon promoter and can upregulate the expression of glucagon-like peptide (GLP-1) (Jin and Liu, 2008), which stimulates glucose-induced insulin secretion and $\beta$-cell proliferation and slows gastric emptying (Grant et al., 2006; Hansson et al., 2010).

Polymorphisms in TCF7L2 may impair its ability to upregulate GLP-1 expression. Human studies have demonstrated that carriers of the risk $\mathrm{T}$ allele showed a weaker response to intravenous glucose and reduced GLP-1 stimulated glucose tolerance in the hyperglycemic state (Grant et al., 2006; Jin and Liu, 2008). Additionally, the risk T allele may impair insulin processing by reducing the conversion of proinsulin to insulin (Loos et al., 2007). Decreased insulin secretion and processing lead to a hyperglycemic state and progression of diabetic retinopathy. Because TCF $7 L 2$ has been hypothesized to influence glycemic control, it may play a role in the development of diabetic retinopathy. In this review, we analyzed clinical studies to better understand the relationship between $T C F 7 L 2$ and diabetic retinopathy. 


\section{MATERIAL AND METHODS}

A systematic literature search of EMBASE, PubMed (MEDLINE), and Scopus was conducted through June 2012 using the MeSH terminology for 'diabetic', 'retinopathy', 'polymorphism', and 'genetic' and adding 'transcription factor 7-like 2' and 'TCF7L2' to the search terms (Supplementary file). A manual search of the references cited in the initially identified articles was also performed. There was no language or study design restriction. Animal studies, in vitro studies, letters, systematic reviews and meta-analyses, examination of a different polymorphism, or articles not containing information about both diabetes retinopathy and TCF7L2 polymorphisms were excluded.

\section{RESULTS}

A total of 69 articles were collected, with 9 duplicate references. After review, 6 clinical studies were identified. One article was dismissed because it was presented as a conference paper; 1 article was not included because the study investigated the entire microsatellite. One article could only be reviewed as an abstract because it was written in Chinese. Therefore, 3 full articles (Melzer et al., 2006; Yan et al., 2010; Buraczynska et al., 2011) and 1 abstract (Fu et al., 2012) were reviewed (Figure 1). A summary of the study results is presented in Table 1. Odd ratios of the risk $T$ allele and a reference genotype $C C$ of TCF7L2 rs7903146 were reported to determine the association between the $\mathrm{T}$ allele and the development of diabetes retinopathy at a significance level of $\mathrm{P}<0.05$.

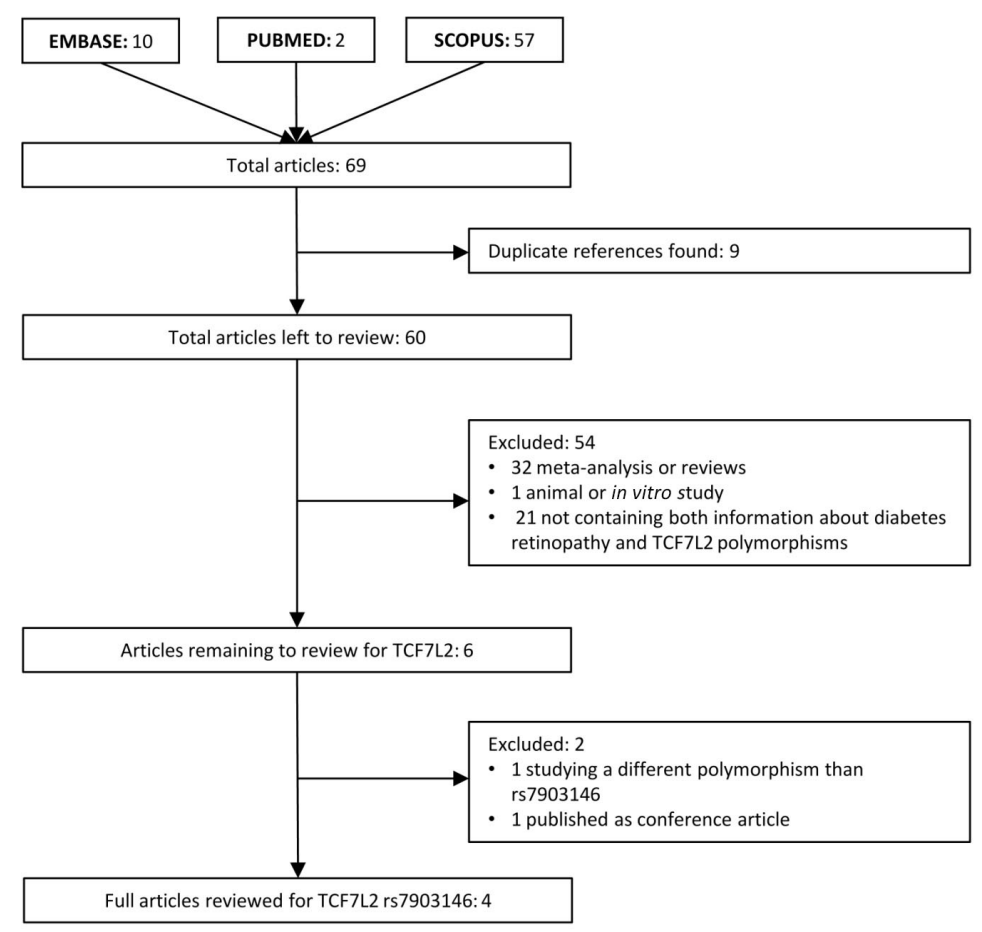

Figure 1. Flowchart of included and excluded studies. 
P. Sudchada and K. Scarpace

5868

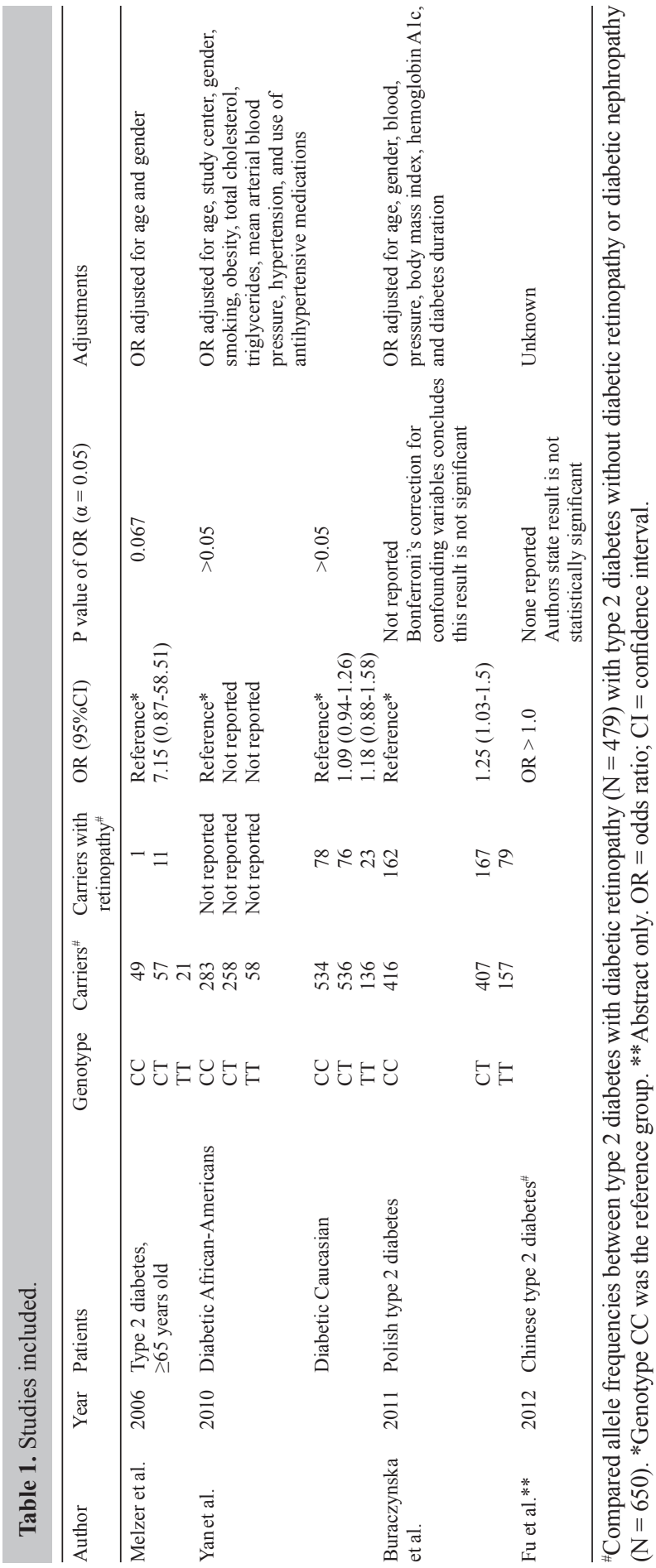


One retrospective case-control study explored the association between TCF7L2 rs7903146 and diabetic biochemical changes and measured diabetic complications between diabetics $(\mathrm{N}=127)$ and non-diabetics $(\mathrm{N}=817)$ using a linear regression model and logtransformed values. The results showed that 12 diabetic subjects had diabetic retinopathy and carried the $\mathrm{T}$ allele, indicating that this allele was not associated with development of diabetes retinopathy (Melzer et al., 2006). However, variation in frequency of the risk T allele was significantly greater in patients with high fasting glucose levels $(\mathrm{P}=0.028)$ (Table 1).

A population-based, longitudinal cohort study explored the association of diabetic retinopathy and the TCF7L2 rs7903146 polymorphism in 2199 African Americans and 8121 American Caucasians. No association was found between the risk $T$ allele and diabetic retinopathy in either African Americans or Caucasians (Yan et al., 2010). These findings were similar to those of a previous study (Melzer et al., 2006) in which subgroup analysis revealed no association between the gene polymorphisms and diabetic retinopathy in diabetic patients (Table 1). Another retrospective case-control study in Polish T2DM subjects (Buraczynska et al., 2011) explored the association between rs 7903146 with T2DM and clinical phenotypes of diabetic manifestations including diabetic retinopathy. Patients with hypertension and normoglycemic patients with diabetic traits were excluded. The study reported that the odds ratios (OR) of the CC genotype vs the risk T allele after adjusting for related factors (Table 1) between the group with $(\mathrm{N}=408)$ or without $(\mathrm{N}=573)$ diabetic retinopathy was $1.25(95 \% \mathrm{CI}=$ 1.03-1.50). However, the result was no longer statistically significant after application of Bonferroni's correction for nephropathy, cardiovascular disease, and early onset diabetes (Table 1). Additionally, the $T C F 7 L 2$ polymorphism was found to be associated with an increased risk of diabetic nephropathy with an OR of $2.98(95 \% \mathrm{CI}=1.16-6.87, \mathrm{~N}=586)$.

A recent retrospective case control study in Asian populations (Fu et al., 2012) investigated the associations between 3 polymorphisms (rs7903146, rs6585205, and rs11196218) and diabetic retinopathy or diabetic nephropathy in T2DM patients. Although the article is in Chinese, the results presented in the English abstract were reviewed. Genotype frequencies of diabetic retinopathy $(\mathrm{N}=479)$ and without diabetic retinopathy $(\mathrm{N}=650)$ were compared. The OR for the association of diabetic retinopathy and the risk rs7903146 T allele was greater than 1 , but this value was not statistically significant $(\mathrm{P}>0.05)$ (Table 1).

\section{DISCUSSION}

TCF7L2 has been shown to be associated with diabetes. However, the relationship between $T C F 7 L 2$ and diabetic retinopathy is not well understood. Four clinical studies that investigated the association between TCF7L2 polymorphisms rs7903146 and diabetic retinopathy were reviewed. Based on the results of these studies, there is no definite and strong correlation between the TCF7L2 polymorphism rs7903146 and diabetic retinopathy.

Limited by its small sample size, the relationship between TCF7L2 rs7903146 polymorphisms and diabetic retinopathy was not observed in the study by Melzer et al. (2006). Participants were recruited from the same Tuscan community in Italy, and it is unclear whether the study participants were representative of the T2DM population, specifically because no standard definition of diabetic retinopathy was used. However, this study found a significant correlation between the frequency of the risk $T$ allele and high fasting glucose levels and supported current research demonstrating a relationship between TCF7L2 polymorphisms and diabetes.

A larger subpopulation study stratified based on ethnicity by Yan et al. (2010) was 
strengthened by its large and diverse population and its specific standard definitions of diabetes and diabetic retinopathy. Similarly to previous study (Melzer et al., 2006), no statistical significant association was observed between the risk $\mathrm{T}$ allele and diabetic retinopathy; the frequency of the risk $\mathrm{T}$ allele was similar in African Americans and Caucasians.

Buraczynska et al. (2011) found an association between TCF7L2 rs7903146 and diabetic retinopathy in Polish type 2 diabetics; the study also revealed a significant relation between the risk $T$ allele and diabetic retinopathy. However, the result was no longer significant after adjusting for confounding variables. The major confounding variables were likely due to diabetic nephropathy, as $408(53 \%)$ patients with diabetic retinopathy also had diabetic nephropathy. Similarly, in the diabetic nephropathy population, 199 (46\%) patients also had retinopathy, but the association of $T C F 7 L 2$ remained statistically significant after adjusting for confounding variables. This result suggests that the risk $\mathrm{T}$ allele may be associated with microvasculature changes and is not specific to retinopathy.

Unfortunately, the most recent study by Fu et al. (2012) was not available in English. It represents the only clinical studies investigating the TCF7L2 polymorphism and diabetic retinopathy in an Asian population. The analysis of this study is limited because the definitions used for diabetes and diabetic retinopathy as well as the inclusion and exclusion criteria are unknown and the authors did not report a specific OR.

One study was excluded from this review (Buchbinder et al., 2008) because the microsatellite DG10S478 was determined rather than the TCF7L2 rs7903146 polymorphism. This study grouped all polymorphisms together as one allele $\mathrm{X}$; the frequency of homozygous $\mathrm{XX}$ and heterozygous $\mathrm{X}$ was compared to the control, homozygous wild-type genotype, 00, in patients with and without microvasculature complications, which included diabetic retinopathy and diabetic nephropathy. However, no significant association between the risk $\mathrm{X}$ allele and microvasculature complications was observed.

Various genes have been studied in diabetic retinopathy, but few have been shown to be strongly associated with the risk of developing diabetic retinopathy. Six genes thought to be associated with diabetic retinopathy have been reviewed in meta-analysis studies (Abhary et al., 2009; Zhou and Yang, 2010). The genes studied can be divided into 2 classes based on the proposed mechanism of involvement in diabetic retinopathy. Genes thought to be associated with direct effects on the retinal microvascular system include angiotension converting enzyme, vascular endothelial growth factor (VEGF), endothelial nitric oxide synthase, and receptor for advanced glycation end products. In addition, genes predicted to be indirectly associated with diabetic retinopathy based on glycemic control include methylenetetrahydrofolate reductase and aldose reductase (Abhary et al., 2009; Zhou and Yang, 2010).

Polymorphisms at intron 16 and an SNP rs3138808 of the angiotension converting enzyme have been hypothesized to be associated with diabetic retinopathy based on the direct actions of angiotensin II on the retina. However, no association was observed for any forms of diabetic retinopathy (Abhary et al., 2009; Zhou and Yang, 2010). VEGF is a cytokine that causes microvascular changes and promotes angiogenesis. It is hypothesized that in the hypoxic state caused by diabetic microvascular changes, VEGF increases vessel permeability, leading to a breakdown of the blood-retinal barrier and neovascularization (Abhary et al., 2009). However, a meta-analysis of 4 VEGF SNPs (rs25648, rs3095039, rs35569394, and rs699947) showed no association with T2DM with all types of diabetic retinopathy across all subpopulations (Zhao and Zhao, 2010). Endothelial nitric oxide synthase is a vasodilator that has been shown to promote angiogenesis and is associated with the regulation of $V E G F$ expression. A 
recent meta-analysis (Zhao et al., 2012) reported inverse associations between polymorphisms of the NOS 3 4b/a intron and T-786C in T2DM subjects (Zhao et al., 2012). When the receptor for advanced glycation end products is activated, it is thought to induce oxidative stress and increase vascular permeability. Based on the results of a meta-analysis (Niu et al., 2012), upon stratification of the population, G1740T SNPs were found to be significantly associated with diabetic retinopathy in diabetics $(\mathrm{OR}=1.24,95 \% \mathrm{CI}=1.01-1.51)$. Methylenetetrahydrofolate reductase catalyzes the methylation of homocysteine to methionine. Hyperhomocysteine levels are associated with hyperglycemia and thus may increase risk of diabetic retinopathy. Carriers of $677 \mathrm{~T}$ showed a 1.32-fold increased risk of developing diabetic retinopathy in relatives of diabetic patients without retinopathy $(95 \% \mathrm{CI}=1.06-1.64, \mathrm{P}=0.011)$ (Niu and Qi, 2012). Multiple polymorphisms in the aldose reductase gene were found to be associated with diabetic retinopathy. Under hyperglycemic conditions, the intracellular concentration of sorbitol increases, which may cause osmotic stress and activate protein kinase $\mathrm{C}$, resulting in pathogenic vascular changes. Supported by 2 meta-analysis, this gene may be associated with the development of diabetes retinopathy (Abhary et al., 2009, 2010).

We found no review articles examining the association between TCF7L2 polymorphisms and diabetic retinopathy. All studies reviewed confirm the relationship between TCF7L2 polymorphisms and diabetes but do not demonstrate a strong association with diabetic retinopathy. Although TCF7L2 polymorphisms are thought to have mainly effects on $\beta$ cells in the pancreas to impair insulin secretion, the strong relationship was not demonstrated. It is likely because the TCF7L2 polymorphisms have indirectly effects on diabetic retinopathy as it does not act directly on the retinal microvascular system. TCF 22 may impair a patient's ability to control serum glucose levels, and chronic hyperglycemia may increase the risk of diabetic retinopathy. This is similar to the mechanism of association of polymorphisms in aldose reductase and methylene tetrahydrofolate reductase. However, genes associated with direct effects on microvasculature structure did not show a significant association with diabetic retinopathy (Abhary et al., 2009; Zhou and Yang, 2010). Because the mechanism of development of diabetic retinopathy are complicated, only analyzing the association between $T C F 7 L 2$ and diabetic retinopathy may not be appropriate. Other potential genes related to diabetic retinopathy should be examined to identify the link between these genes and diabetes retinopathy.

In conclusion, the limitations of previous studies make it difficult to draw conclusions regarding the association between TCF7L2 rs7903146 and diabetic retinopathy. Based on the results of the 4 studies reviewed here, the association between $T C F 7 L 2$ and diabetic retinopathy is complex. Polymorphisms in TCF7L2 rs7903146 likely play a role in the genetics, placing patients at risk for developing diabetic retinopathy. Further studies involving larger, ethnically diverse populations and adjusting for confounding variables to determine the relationship among TCF7L2 rs7903146 and other potential genes with diabetic retinopathy are warranted.

\section{Supplementary material}

\section{REFFERENCES}

Abhary S, Hewitt AW, Burdon KP and Craig JE (2009). A systematic meta-analysis of genetic association studies for diabetic retinopathy. Diabetes 58: 2137-2147.

Abhary S, Burdon KP, Laurie KJ, Thorpe S, et al. (2010). Aldose reductase gene polymorphisms and diabetic retinopathy 
susceptibility. Diabetes Care 33: 1834-1836.

Buchbinder S, Rudofsky G Jr, Humpert PM, Schilling T, et al. (2008). The DG10S478 variant in the TCF7L2 gene is not associated with microvascular complications in type 2 diabetes. Exp. Clin. Endocrinol. Diabetes 116: 211-214.

Buraczynska M, Swatowski A, Markowska-Gosik D, Kuczmaszewska A, et al. (2011). Transcription factor 7-like 2 (TCF7L2) gene polymorphism and complication/comorbidity profile in type 2 diabetes patients. Diabetes Res. Clin. Pract. 93: 390-395.

Cauchi S and Froguel P (2008). TCF7L2 genetic defect and type 2 diabetes. Curr. Diab. Rep. 8: 149-155.

Fu LL, Lin Y, Yang ZL and Yin YB (2012). Association analysis of genetic polymorphisms of TCF7L2, CDKAL1, SLC30A8, HHEX genes and microvascular complications of type 2 diabetes mellitus. Zhonghua Yi Xue Yi Chuan Xue Za Zhi. 29: 194-199.

Grant SF, Thorleifsson G, Reynisdottir I, Benediktsson R, et al. (2006). Variant of transcription factor 7-like 2 (TCF7L2) gene confers risk of type 2 diabetes. Nat. Genet. 38: 320-323.

Hansson O, Zhou Y, Renstrom E and Osmark P (2010). Molecular function of TCF7L2: Consequences of TCF7L2 splicing for molecular function and risk for type 2 diabetes. Curr. Diab. Rep. 10: 444-451.

Helgason A, Palsson S, Thorleifsson G, Grant SF, et al. (2007). Refining the impact of TCF7L2 gene variants on type 2 diabetes and adaptive evolution. Nat. Genet. 39: 218-225.

Jin T and Liu L (2008). The Wnt signaling pathway effector TCF7L2 and type 2 diabetes mellitus. Mol. Endocrinol. 22: 2383-2392.

Loos RJ, Franks PW, Francis RW, Barroso I, et al. (2007). TCF7L2 polymorphisms modulate proinsulin levels and betacell function in a British Europid population. Diabetes 56: 1943-1947.

Lyssenko V (2008). The transcription factor 7-like 2 gene and increased risk of type 2 diabetes: an update. Curr. Opin. Clin. Nutr. Metab. Care 11: 385-392.

Melzer D, Murray A, Hurst AJ, Weedon MN, et al. (2006). Effects of the diabetes linked TCF7L2 polymorphism in a representative older population. BMC Med. 4: 34 .

Niu W and Qi Y (2012). An updated meta-analysis of methylenetetrahydrofolate reductase gene 677C/T polymorphism with diabetic nephropathy and diabetic retinopathy. Diabetes Res. Clin. Pract. 95: 110-118.

Niu W, Qi Y, Wu Z, Liu Y, et al. (2012). A meta-analysis of receptor for advanced glycation end products gene: four wellevaluated polymorphisms with diabetes mellitus. Mol. Cell Endocrinol. 358: 9-17.

Shu L, Sauter NS, Schulthess FT, Matveyenko AV, et al. (2008). Transcription factor 7-like 2 regulates beta-cell survival and function in human pancreatic islets. Diabetes 57: 645-653.

Tong Y, Lin Y, Zhang Y, Yang J, et al. (2009). Association between TCF7L2 gene polymorphisms and susceptibility to type 2 diabetes mellitus: a large Human Genome Epidemiology (HuGE) review and meta-analysis. BMC Med. Genet. 10: 15.

Weedon MN (2007). The importance of TCF7L2. Diabet. Med. 24: 1062-1066.

Yan Y, Klein R, Heiss G, Girman CJ, et al. (2010). The transcription factor 7-like 2 (TCF7L2) polymorphism may be associated with focal arteriolar narrowing in Caucasians with hypertension or without diabetes: the ARIC Study. BMC Endocr. Disord. 10: 9.

Yau JW, Rogers SL, Kawasaki R, Lamoureux EL, et al. (2012). Global prevalence and major risk factors of diabetic retinopathy. Diabetes Care 35: 556-564.

Zhao S, Li T, Zheng B and Zheng Z (2012). Nitric oxide synthase 3 (NOS3) 4b/a, T-786C and G894T polymorphisms in association with diabetic retinopathy susceptibility: a meta-analysis. Ophthalmic Genet. 33: 200-207.

Zhao $\mathrm{T}$ and Zhao J (2010). Association between the $-634 \mathrm{C} / \mathrm{G}$ polymorphisms of the vascular endothelial growth factor and retinopathy in type 2 diabetes: a meta-analysis. Diabetes Res. Clin. Pract. 90: 45-53.

Zhou JB and Yang JK (2010). Angiotensin-converting enzyme gene polymorphism is associated with proliferative diabetic retinopathy: a meta-analysis. Acta Diabetol. 47 (Suppl 1): 187-193.

Zimmet P, Alberti KG and Shaw J (2001). Global and societal implications of the diabetes epidemic. Nature 414: 782-787. 